\title{
$X X X$. On the figure of equilibrium of a homogeneous planet in a fluid state; in reply to the observations of M. Poisson, published in this Journal for July last
}

\author{
James Ivory Esq. M.A. F.R.S.
}

To cite this article: James Ivory Esq. M.A. F.R.S. (1827) XXX. On the figure of equilibrium of a homogeneous planet in a fluid state; in reply to the observations of M. Poisson, published in this Journal for July last, Philosophical Magazine Series 2, 2:9, 161-168, DOI: 10.1080/14786442708675640

To link to this article: http://dx.doi.org/10.1080/14786442708675640

曲 Published online: 10 Jul 2009.

Submit your article to this journal ¿

Џll Article views: 2

Q View related articles $₫$ 


\title{
PHILOSOPHICAL MAGAZINE
}

A N D

\section{ANNALS OF PHILOSOPHY. \\ [NEW SERIES.]}

\author{
$S E P T E M B E R$ 1827.
}

XXX. On the Figure of Equilibrium of a Homogeneous Planet in a Fluid State; in reply to the Observations of M. Poisson, published in this Journal for July last. By J. Ivory, Esq. M.A. F.R.S.*

THE two letters which I have addressed to Professor Airy in the last Numbers of this Journal, fully ascertain the nature and extent of the analytical method employed by Laplace in the investigation of the figure of the planets. I now proceed to the conditions which I have found to be necessary for solving the problem of a homogeneous mass of fluid revolving upon an axis, to which M. Poisson objects. The subject is one of considerable importance; it involves a capital question in the system of Newton, towards the solution of which nothing has in reality been added since the time of Maclaurin and Clairaut. This is the more unaccountable, because, in the long interval elapsed, the problem has continued to occupy the attention of all the great mathematicians; the difficulties attending the computation of the attractive forces, which embarrassed the first inquirers, have been overcome by the progress of mathematical science; and we are in possession of a theory of the equilibrium of fluids, which is supposed to be exact and complete. Yet these accessions to our knowledge have been made not only without advancing the problem of the figure of the planets, but even without reaching what had previously been investigated by a more simple geometry. What reasons can be assigned for this total ineffciency? Any attempt to elucidate this matter, and to detect

* Communicated by the Author.

New Series. Vol. 2. No. 9. Sept. 1827. 
any misapprehension or error that may have impeded the progress of this branch of mechanical philosophy, is at least deserving of serious attention, and ought not to be fastidiously thrown aside merely because it is opposed to what has received the sanction of great names.

In what follows, homogeneous fluids only are understood; and it is supposed that the accelerating forces which urge the particles, are expressed by functions of the coordinates that satisfy the criterion of integrability. It will also be proper to notice a distinction depending on the nature of the accelerating forces, inattention to which has been the cause of no little perplexity in this theory. The forces mentioned may be either explicitly given, so as to be entirely and absolutely known when we know the coordinates of a molecule of the fluid upon which they act; or the same forces may be relative to the unknown figure of the mass of fluid.

If the particles of a fluid are urged by attractions to fixed centres and by centrifugal forces, the problem of equilibrium falls under the first division. In such cases all the level surfaces have the same differential equation, and they are different only in the different pressures they sustain. When any one of those surfaces is known, all the rest are derived from it merely by varying the pressure, and the figure of the whole mass in equilibrio, is necessarily determined. At the outer surface of the fluid there is no pressure; and, therefore, when the outer surface satisfies the differential equation common to all the level surfaces, or, which is the same thing, when the resultant of the accelerating forces urging the particles is perpendicular to that surface, all that is required for solving the problem is fulfilled.

When the particles of a fluid attract one another, the accelerating forces urging them will depend upon the place they occupy and upon the figure of the whole mass. As the attractive forces upon points differently situated must vary, all the level surfaces will not, in this case as before, have the same differential equation. These surfaces will now vary from one another on two accounts; namely, the different pressures they sustain, and the variation of the attractive forces according to the situation of the surfaces in the mass. It appears, therefore, impossible in this case to establish any general relation between the several level surfaces without the special consideration of the figure of the fluid. We cannot infer that all the level surfaces will exist in the interior of the fluid, and will satisfy, each its peculiar equation, merely because gravity acts in directions perpendicular to the outer surface. There 
appears to be no absurdity in supposing that the latter condition may be fulfilled, at the same time that no level surfaces exist in the interior, and consequently when there is no equilibrium of the mass of fluid.

It has been shown that there is a plain distinction between a fluid in equilibrio when the accelerating forces acting upon the particles are explicitly given, and when the same forces depend upon the figure of the mass. Yet, according to the received theory there is no difference in the two cases with respect to the conditions necessary to insure the equilibrium. Supposing that the algebraic expressions of the forces possess the criterion of integrability, nothing further is required in either case, than that the outer surface of the fluid be a level surface cutting the direction of gravity everywhere at right angles. But if the determination of the equilibrium be the same in both cases, we might expect that the demonstration would be different in circumstances so essentially distinguished. It must be confessed, however, that we meet with nothing in the shape of demonstration, except what is vague and unsatisfactory and applies alike to both cases. The principle seems to be this: granting that the exterior surface is a level surface, it is always possible to trace the level surfaces in the interior; because, since there is no distinction of density, we may adopt any surfaces we please as level surfaces*. When the accelerating forces are explicitly given, there is no difficulty nor ambiguity; because the equation of all the level surfaces are nowise different except in the constants introduced by integration, which vary from one surface to another. But the case is not the same when the level surfaces depend upon the figure of the fluid. Taking a point in the interior of the mass, we may indeed conceive a surface to pass through it and to be extended on all sides, so as everywhere to cut the resultant of the accelerating forces at right angles, and we may call this a level surface: but we may thus fall into error; because it is not clear that the surface so traced will return into itself and completely inclose a portion of the fluid; and without this it would neither be a level surface, nor would there be an equilibrium of the fluid. In order to place the theory on a solid foundation, such gratuitous assumptions must be set aside; and it must be proved, by means of the equations which accurately determine the level surfaces, that they do necessarily exist in the interior of the fluid, and produce the equilibrium in question. But this has not been done; and, to say

* Mécan. Céleste, Liv. $1^{\text {re }}$, No. $17 ; \&$ Liv. $3^{\text {me }}$, No. 22. 
the least, it is very doubtful whether it can possibly be accomplished.

Being dissatisfied with the usual equations for finding the figure of a homogeneous planet in a fluid state, I have sought to deduce a solution of the problem by a strict analysis, without admitting any gratuitous assumption. By analysis I do not here mean an algebraic calculation; I understand by it a process of reasoning such as the ancient geometers employed to derive the construction of a geometrical problem from the conditions to be fulfilled. In the following investigation I confine my attention to a homogeneous planet in a fluid state; that is, to the equilibrium of a fluid mass of uniform density, the particles attracting one another inversely as the square of the distance, and being urged by a centrifugal force caused by rotation about an axis that passes through the centre of gravity; and in order that the train of reasorting may be more easily examined, I divide it in distinct propositions.

Prop. 1.-If two particles be similarly placed, in two bodies, exactly similar in their figure and composed of the same homogeneous matter; the attractive forces of the bodies upon the particles will act in similar directions, and will be proportional to the linear dimensions of the bodies.

Particles here mean infinitely small portions of the two bodies proportional to the whole masses. The proposition is proved, Prin. Math. lib. i. Prop. 87.-Maclaurin's Fluxions, $\$ 629$.

Prop. 2.-If a homogeneous mass of fluid revolving upon an axis be in equilibrio by the attraction of its particles in the inverse proportion of the square of the distance; any other mass of the same fluid having a similar figure and revolving with the same rotatory velocity about an axis similarly placed, will likewise be in equilibrio, supposing that its particles attract one another by the same law.

Take two particles similarly situated in the two bodies. By Prop. 1. the resultants of the attractive forces acting on the particles have similar directions, and are proportional to the linear dimensions of the bodies. Further, the centrifugal forces urging the particles to recede from the axes of rotation, are proportional to the respective distances from those axes, that is, to the linear dimensions of the bodies. Wherefore the joint effect of all the forces is to urge the particles in similar directions with intensities proportional to the linear dimensions of the bodies. And as the same thing is true of all particles similarly situated in the two bodies, it follows that if there be an equiliorium in one case, there will likewise be an equilibrium 
equilibrium in the other: for the forces which urge the particles of one body are different in no respect from the forces which urge the particles of the other, except in being all increased, or all diminished, in the same given proportion *.

Prop. 3.-If a homogeneous mass of fluid revolve about an axis which passes through the centre of gravity, and be in equilibrio by the attraction of its particles in the inverse proportion of the square of the distance; any surface in the interior, similar to the outer surface and similarly posited about the centre of gravity, will be a level surface.

For, by the hypothesis, the whole mass of fluid and the portion of it bounded by the interior surface are similar in their figures; and they both revolve in the same time about the common axis which cuts them similarly: wherefore the first of these two bodies being in equilibrio, it follows from the last proposition, that the other would likewise be in equilibrio if it revolved by itself, the exterior matter being taken away or annihilated. Thus the interior fluid body is in equilibrio in two different states : first, when it revolves by itself, in which case the only forces in action are, the attraction of its particles and their centrifugal force; and secondly, when it is a part of the whole fluid mass, in which case there is superadded to the former forces, the action of all the exterior matter. Now these two states of equilibrium cannot consist with one another, if we suppose that the exterior matter has any other effect than to produce an equable pressure upon the surface of the interior body; that is, unless the same surface be a level surface.

- In the Annales de Physique et de Chimie, tom. xxvii. p. 234, M. Poisson makes use of a very curious argument with respect to this proposition: - "Proposition dont l'inverse ne serait pas vraie; car on sait que pour une même densité et une même vitesse de rotation, qui ne dépasse pas une certaine limite, il y a deux ellipsoïdes dissemblables qui satisfont à l'equilibre d'une masse fluide." Now all this is quite beside the purpose. There is no question about inverse propositions. The theorem as I have laid it down is undoubtedly true; and it may therefore be legitimately used in deducing from the existence of the equilibrium the essential properties that belong to it, and without which it cannot subsist. M. Poisson, not adverting that my reasoning is as much an analysis as if it had been expressed by algebraic equations, goes on to say, that the new condition is not necessary to the equilibrium. Now I have here proved that the equilibrium cannot take place without it, and that the usual condition is by itself insufficient.

The argument in p. 235 is equally inapplicable. The case mentioned is one in which the accelerating forces urging the particles is independent of the figure of the fluid: it is one in which the forces are explicitly given, and is therefore attended with no difficulty.

By means of such loose argumentation any point may be proved or disproved at pleasure. 
The foregoing propositions, which have been deduced by analytical reasoning from the single hypothesis that there is an equilibrium, contain essential properties, without which the equilibrium cannot subsist in any possible figure of the fluid mass. Another property is now to be added, no less essential, no less independent of all considerations relative to a particular figure, and equivalent to the new condition employed for finding the figure of a homogeneous planet, to which M. Poisson has objected.

Prop. 4.-Suppose that a homogeneous mass of fluid, revolving about an axis which passes through the centre of gravity, is in equilibrio by the attraction of its particles in the inverse proportion of the square of the distance; take any point in the interior of the fluid, the distance of which from the centre of gravity is equal to $r$, and let $V(r)$ denote the sum of all the molecules of the whole mass divided by their respective distances from the assumed point, and $V^{\prime}(r)$ the like sum extending only to all the molecules within the level surface passing through the same point: then is $V(r)-V^{\prime}(r)$ a constant quantity for all points of the same level surface.

Let $\phi$ denote the centrifugal force at the distance ' from the axis of rotation, and $\theta$ the angle which $r$ makes with the same axis : then, as is well known to all geometers, thee quation of the interior level surface will be,

$$
\mathrm{V}(r)+\phi \times \frac{r^{2}}{2} \sin ^{2} \theta=\mathrm{C}
$$

$C$ being constant for all points of the same level surface, and varying from one level surface to another. But, according to the second and third propositions, the portion of fluid bounded by the interior level surface would be in equilibrio without any change of its figure, if it revolved by itself; in which case the equation of the surface would be,

$$
\mathrm{V}^{\prime}(r)+\Phi \times \frac{r^{2}}{2} \sin ^{2} \theta=\mathrm{C}^{\prime}
$$

Wherefore, by subtracting the two equations, we get,

$$
\mathrm{V}(r)-\mathrm{V}^{\prime}(r)=\mathrm{C}-\mathrm{C}^{\prime}
$$

which is the property to be proved, because $\mathbf{C}$ and $\mathbf{C}^{\prime}$ are the same for all points of the same level surface.

The property just investigated and the equation of the outer surface of the fluid are together sufficient for determining the figure of a homogeneous planet. But without the property mentioned, the equilibrium would not take place. For nothing followsfrom the equation of the outer surface of the fluid alone, except that the equation (2) would be true of all the interior surfaces similar to the outer one and similarly posited about 
the centre of gravity. Now these surfaces are independent of all the matter exterior to them; and therefore they are not level surfaces, and the mass of fluid is not in equilibrio. But when the equations (2) and (3) exist at the same time, we thence derive the equation (1), which is the true equation of the level surfaces, and establishes the equilibrium.

It appears that $V(r)-V^{\prime}(r)$ is the pressure upon the assumed point in the interior of the fluid. The pressure therefore is wholly determined by the figure of equilibrium, and has no immediate dependence upon the centrifugal force; and it is easy to see that this must be the case. For there is no centrifugal force at the points where the axis of rotation traverses the several level surfaces : at these points, therefore, the centrifugal force has no influence on the pressure; and, as the pressure is the same over the whole of every level surface, it follows that it is independent of the centrifugal force in all the interior of the fluid.

The similarity of the interior surfaces and the property contained in the fourth proposition, determine the figures with which the equilibrium is possible; and it is found that they can only be ellipsoids : the equation of the outer surface ascertains the rotatory velocity that must take place in every particular figure. We are therefore entitled, upon undoubted evidence, to establish the following proposition:

Prop. 5.-A homogeneous mass of fluid consisting of particles that attract one another inversely as the square of the distance, and revolving upon an axis passing through the centre of gravity, cannot be in equilibrio nor maintain a permanent figure, unless it have the figure of an ellipsoid.

The whole of the preceding reasoning may be brought within a narrow compass. If a homogeneous planet in a fluid state be in equilibrio, all the level surfaces must be similar to one another: but the ellipsoid is the only figure in which the level surfaces are all similar; wherefore the planet must have the figure of an ellipsoid. All this, it is presumed, is here proved by exact reasoning. On the other hand, the usual theory is grounded on an assumption without proof. If the outer surface of a homogeneous mass of fluid be a level surface, it is assumed, for no other reason than that there is no distinction of density, that all the level surfaces in the interior do necessarily exist and produce an equilibrium. But, if there be no distinction of density, there is a general equation belonging to the interior level surfaces; and, in order to prove the existence of these surfaces, on which the equilibrium depends, it should be shown that their equation is a necessary consequence of that of the outer surface. But this has not 
been done, except in the case when the accelerating forces urging the particles are explicitly given.

The foregoing solution of the problem is essentially the same with that contained in my paper, Phil. Trans. 1824. M. Poisson has objected to my conditions of equilibrium in an article printed in the Annales de Physique et de Chimie, tom. xxvii. p. 225, which I have not examined till very lately, since the publication of his remarks in this Journal for July last. If his objections have not been noticed here, care has been taken to place the subject in such a point of view as to avoid their force. Of his arguments, when they are not chargeable with insufficient reasoning,--some do not apply to my theory, and some are' not inconsistent with it. But the length of what $I$ have written obliges me to postpone my further remarks on this subject to a future occasion.

Aug. 6, 1827.

JAMES Ivory.

[To be continued.]

XXXI. Collections in Foreign Geology.-[No. III.] By H. 'T. De la Beche, Esq. F.R., L., and G.S. \&c. \&c.

[Continued from page 109.]

\section{Introduction to the Mineral Geography of Sroeden; by} M. Hisinger*.

THE Scandinavian Peninsula, extending from S.S.W. to N.N.E. from the southern point of Scania to Cape North, is cut into a multitude of gulfs (Fiordar) on its western side, and traversed by a long chain of mountains (Fiellrygg) from Lindesnæs in Norway to the Frozen Ocean, approaching nearer to the North Sea than the Baltic; so that its western side is very steep, whilst towards Sweden the land falls in a very gradual manner. The southern part of this chain is named Langfield; the middle portion Dovrefield. The latter obliquely cuts the Peninsula, and is prolonged to above lake Oresund, at the point where the Herjedal and Jemtland abut on Norway. The Scandinavian Alps may thus be divided into three or four portions, which are united in such a man-

* From a notice in Baron de Férussac's Bulletin des Sciences, for March 1826, of a German translation in Leonhard's Zeitschrift für Mineralogie.

For excellent descriptions of the primitive rocks of the northern part of our island, which (with similar rocks in Ireland) may be considered as the geological prolongation of the Scandinavian Peninsula, consult Dr. Macculloch's Western Islands, Classification of Rocks, and papers in the Geological Transactions. For those of the E. of Ireland, the memoir of Mr. Weaver, in the Geol. Transactions.-Trans. 